\title{
La douleur est sexiste !
}

\author{
S. Marchand \\ (C) Springer-Verlag 2009
}

Il est évident qu'il existe des différences de prévalence pour certaines maladies entre les hommes et les femmes. Il en va de même pour la douleur. Les études épidémiologiques supportent que les femmes soient surreprésentées dans l'apparition et la persistance de la douleur. Pourtant, ces différences entre les sexes n'apparaissent qu'à la puberté, ce qui laisse présager un rôle important des hormones sexuelles. Il en va de même pour la réponse au traitement, la réponse analgésique à des approches pharmacologiques comme les opioïdes n'est pas identique entre les femmes et les hommes. De plus, les réponses physiologiques, comme celle du système nerveux autonome, présentent aussi des différentes significatives selon le sexe. Les hommes présentent des réponses sympathiques dès le début de la douleur, tandis que les femmes vont plus rapidement présenter des réponses parasympathiques, ce qui change les réponses du système autonome, comme la fréquence cardiaque qui est parfois un indice utilisé pour mesurer la douleur chez les patients non communiquant, indépendamment du sexe de l'individu.

Il est intéressant de souligner que ces différences dans la douleur entre les sexes existent, mais il faut aussi tenter d'en comprendre les mécanismes. C'est ce que tente de faire les auteurs de ce numéro en publiant des articles qui mettent à jour nos connaissances sur les bases biologiques et physiologiques des différences de perception, de prévalence et de réponse au traitement de la douleur entre les femmes et les hommes.

Dans l'article de Souza et al., une étude populationnelle fait bien ressortir l'importante prévalence de la douleur chronique chez les femmes en tenant compte de facteurs tels que l'âge. Cet article met aussi en lumière les différences de la perception de leur santé qu'ont les femmes et les hommes.

Dans leur article, Aloisi et al. met à jour des données qualitatives et quantitatives qui proposent des pistes pour mieux comprendre les mécanismes impliqués dans les différences selon le sexe dans la douleur. Ils mettent en

S. Marchand $(\bowtie)$

Faculté de médecine, neurochirurgie, 3001, $12^{\mathrm{e}}$ avenue Nord,

Sherbrooke, Québec J1H 5N4, Canada

e-mail : serge.marchand@usherbrooke.ca relation autant des études fondamentales chez l'animal que des études chez l'humain pour une meilleure compréhension de ces mécanismes.

L'article de Dr Gaumond met une emphase particulière sur le rôle des hormones sexuelles dans les différences dans la douleur selon le sexe. Elle fait une excellente synthèse de l'importance du rôle des mécanismes excitateurs, mais aussi des mécanismes inhibiteurs endogènes de la douleur et comment les hormones sexuelles modulent ces mécanismes.

Dr Tousignant-Laflamme écrit un article de synthèse sur l'importance du système nerveux autonome dans les différences selon le sexe dans la douleur. Il nous informe ainsi que les réponses autonomiques ne sont pas les mêmes entre les deux sexes et que ces différences peuvent avoir des conséquences pour l'évaluation de la douleur et même mener à des iniquités entre les sexes.

Drs Keller et Poisbeau font une excellente revue des connaissances actuelles sur le rôle des neurostéroïdes, ces hormones qui peuvent être synthétisés directement par les cellules du système nerveux central, dans la douleur. Les neurostéroïdes jouent un rôle très important dans les différences entre les sexes et permettent aussi de comprendre pourquoi mesurer uniquement les hormones circulantes ne permet pas toujours d'expliquer les différences de réponses que l'on retrouve entre les femmes et les hommes. Ils proposent aussi de nouvelles avenues thérapeutiques pour le traitement de la douleur.

Finalement, dans leur article, Paul-Savoie et al. s'intéresse au rôle des hormones sexuelles dans un symptôme de douleur particulièrement prévalent chez la femme, la fibromyalgie. Dans cet article, ils synthétisent les nouvelles données sur le rôle des hormones sexuelles dans ce syndrome de douleur complexe.

Ce numéro spécial sur les différences entre les femmes et les hommes dans la douleur n'a pas uniquement pour but de souligner l'importance de reconnaître ces différences, mais propose aussi une revue des mécanismes impliqués et des pistes pour adapter les traitements. Nous espérons ainsi pouvoir participer à une évolution de la pratique du traitement de la douleur qui tiendra compte du sexe dans ses approches thérapeutiques et ainsi adapter adéquatement le traitement. 